\title{
A B-spline finite element method for nonlinear differential equations describing crystal surface growth with variable coefficient
}

\author{
Dandan Qin ${ }^{1,2 *}$, Yanwei Du' , Bo Liu ${ }^{1}$ and Wenzhu Huang ${ }^{3}$
}

"Correspondence:

qdandan66@163.com

${ }^{1}$ College of Mathematics, Jilin

University, Changchun, P.R. China

${ }^{2}$ Fundamental Department,

Aviation University of Air Force,

Changchun, P.R. China

Full list of author information is

available at the end of the article

\begin{abstract}
In this paper, an efficient finite element scheme is presented for a class of fourth-order nonlinear parabolic problems with variable coefficient. To deal with second-order term in weak formulation, we choose the cubic B-spline function as a trial function. Rigorous error estimates are derived for both semi-discrete and fully-discrete schemes. We provide a numerical example to confirm our theoretical results.
\end{abstract}

Keywords: Cubic B-spline; Finite element method; Nonlinear parabolic equation; Variable coefficient; Error estimate

\section{Introduction}

Molecular beam epitaxy (MBE) is a widely practiced technique for depositing atoms from a vapor phase onto a surface. The technique is very important in growing thin films [1,2]. Crystal surface growth has recently received increasing interest in materials science [3, 4]. A reason is that it could be used in the design of semi-conductors.

In the analysis of $\mathrm{MBE}, H(x, t)$ is the hight of the surface above the substrate plane, and $H(x, t)$ satisfies the current continuity equation

$$
H_{t}+\nabla \cdot J_{\text {surface }}\{H\}=F,
$$

where $F$ is the incident mass flux out of the molecular beam. $J_{\text {surface }}$, which depends on the whole surface configuration, is the systematic current. Keeping only the most important terms in a gradient expansion, subtracting the mean height $H=F u$, and using appropriately rescaled units of height, distance, and time [5], one can get

$$
u_{t}=-\gamma \Delta^{2} u-\mu \nabla \cdot\left[f\left(\nabla u^{2}\right) \nabla u\right]
$$

where $\gamma$ and $\mu$ are two positive constants. In equation (2), the linear term models relaxation through adatom diffusion driven by the surface free energy [6], while the nonlinear term simulates the nonequilibrium current [7]. A Burton-Cabrera-Frank type theory [8,

(c) The Author(s) 2019. This article is distributed under the terms of the Creative Commons Attribution 4.0 International License (http://creativecommons.org/licenses/by/4.0/), which permits unrestricted use, distribution, and reproduction in any medium, provided you give appropriate credit to the original author(s) and the source, provide a link to the Creative Commons license, and indicate if changes were made. 
9] indicates the formula $f\left(s^{2}\right)=\frac{1}{1+s^{2}}$ [5]. Hence, we attain the following form:

$$
u_{t}+\gamma \Delta^{2} u+\mu \nabla \cdot\left(\frac{\nabla u}{1+|\nabla u|^{2}}\right)=0, \quad(x, t) \in \Omega \times(0, T)
$$

where $\Omega \subset \mathbb{R}^{2}$ is a bounded domain with smooth boundary.

In [10], Grasselli et al. showed that equation (3) endowed with no-flux boundary conditions generates a dissipative dynamical system under very general assumptions on $\partial \Omega$ on a phase-space of $L^{2}$ type. In the limit of weak desorption, Pierre-Louis et al. derived a 1D case of equation (3) for vicinal surface growing in the step flow mode [11]. This limit turned out to be singular, and nonlinearities of arbitrary order need to be taken into account. For $1 \mathrm{D}$ case of equation (3), Zhao et al. proved that the Hermite finite element method has the convergence rate of $O\left(\Delta t+h^{3}\right)$ (see [12]).

The finite element method (FEM) [13-18], as a type of an important numerical tool for solving differential equations, has a long history. For 1D Cahn-Hilliard equation, Zhang derived the error estimates of FEM [19]. Elliott and French [20] studied a continuous in-time finite-element Galerkin approximation for two-dimensional Cahn-Hilliard equation. In [21], Barrett et al. discussed the FEM for a fourth-order nonlinear degenerate parabolic equation. An iterative scheme, which solves the resulting nonlinear discrete system, is analyzed. In [22], Kästner et al. considered a numerical convergence study of the Cahn-Hilliard phase-field model within an isogeometric finite element analysis framework.

We also want to introduce the development history of the B-spline method. In 1946, the B-spline method was first presented in Schoenberg's paper [23]. Curry and Schoenberg introduced one element B-spline functions in 1966 (see [24]). In 1976, de Boor extended Bspline to multiple situations [25]. Box-splines [26], simple splines [27], and conical splines [28], which were presented by de Boor, Micchelli, and Dahmen respectively, are the elegant forms of different functional forms of the multivariate B-splines. B-splines have been widely used in various fields of numerical analysis [29-31]. Hall studied the cubic B-spline interpolation [32, 33]. There are several papers which study the B-spline FEM [34-39].

B-splines have better smoothness than the Hermite type elements, for example, quadratic B-splines are in $C^{1}(-\infty,+\infty)$. Unfortunately, the convergence order of the FEM using quadratic B-splines is lower. Besides, Hermite type elements have two types of different basis functions, but the B-spline finite element only has one type of basis functions. So we consider the cubic B-spline FEM for a fourth-order nonlinear parabolic equation with variable coefficient. The convergence order of the B-spline FEM is equal to that of the Hermite FEM in paper [12].

The outline of this paper is as follows. In the next section, some basic definitions and results are introduced. In Sect. 3, we study the semi-discrete approximation and derive its convergence rate. In Sect. 4, the error estimate of the fully-discrete scheme is discussed. In Sect. 5, some numerical experiments are presented to confirm our theoretical results.

Throughout this paper, the letters $C$ and $C^{\prime}$ denote generic constants independent of the division size not necessarily the same at different occurrences. On the other hand, we denote $L^{2}, L^{\infty}, H^{k}$ norms in $I$ by $\|\cdot\|,|\cdot|_{\infty}$, and $\|\cdot\|_{k}$, respectively. 


\section{Preliminaries}

For a 1D case of equation (3), considering the fourth-order term with the variable coefficient and the suitable initial and boundary value conditions, we obtain the following problem:

$$
\left\{\begin{array}{l}
u_{t}+\left(\alpha(x, t) u_{x x}\right)_{x x}+\mu\left(\frac{u_{x}}{1+\left|u_{x}\right|^{2}}\right)_{x}=0, \quad(x, t) \in I \times(0, T) \\
u(x, t)=u_{x}(x, t)=0, \quad(x, t) \in \partial I \times(0, T) \\
u(x, 0)=u_{0}(x), \quad x \in I
\end{array}\right.
$$

where $I=[0,1]$. The variable coefficient $\alpha(x, t)$ satisfies the assumption

$$
\alpha(x, t), \frac{\partial \alpha}{\partial t}(x, t) \in C(I \times[0, T]),
$$

and there exist three positive constants $s, S$, and $M$ such that

$$
\begin{aligned}
& 0<s \leq \alpha(x, t) \leq S<+\infty, \quad(x, t) \in I \times[0, T] \\
& \left|\frac{\partial \alpha}{\partial t}\right| \leq M, \quad(x, t) \in I \times[0, T] .
\end{aligned}
$$

Remark 2.1 Notice that the essential boundary conditions are $u(0, t)=u(1, t)=u_{x}(0, t)=$ $u_{x}(1, t)=0$. Then we define the following space:

$$
H_{0}^{2}(I)=\left\{w ; w \in H^{2}(I), w(0, t)=w(1, t)=w_{x}(0, t)=w_{x}(1, t)=0\right\} .
$$

Let $k$ be any positive integer and $\varphi_{k}(x)$ denote the $k$ th-order B-spline with knots at the set $\mathbb{Z}$ of integers such that $\operatorname{supp}\left(\varphi_{k}\right)=[0, k]$. More precisely, $\varphi_{k}(x)$ is defined recursively by

$$
N_{k}(x)=\left(N_{k-1} * N_{1}\right)(x)=\int_{0}^{1} N_{k-1}(x-t) d t
$$

with

$$
N_{1}(x)=\chi_{[0,1]}= \begin{cases}1, & x \in[0,1] \\ 0, & \text { else }\end{cases}
$$

$N_{4}(x)$ is obviously the cubic B-spline and can be expressed as

$$
N_{4}(x)= \begin{cases}\frac{1}{6} x^{3}, & x \in[0,1], \\ -\frac{1}{2} x^{3}+2 x^{2}-2 x+\frac{2}{3}, & x \in[1,2], \\ \frac{1}{2} x^{3}-4 x^{2}+10 x-\frac{22}{3}, & x \in[2,3], \\ -\frac{1}{6}(x-4)^{3}, & x \in[3,4], \\ 0, & \text { else. }\end{cases}
$$

It is known that the B-spline $N_{k}(x)$ is in $C^{k-2}(-\infty, \infty)$. 
The variational problem related to (4) is: Find $u=u(\cdot, t) \in H_{0}^{2}(I)(0 \leq t \leq T)$ such that

$$
\left\{\begin{array}{l}
\left(u_{t}, v\right)+\left(\alpha(x, t) D^{2} u, D^{2} v\right)=\mu\left(\frac{D u}{1+|D u|^{2}}, D v\right), \quad \forall v \in H_{0}^{2}(I), \\
u(x, 0)=u_{0}(x), \quad x \in I
\end{array}\right.
$$

where $D u=\frac{\partial u}{\partial x}$. We give the existence of the solution of problem (8) in the following theorem (see [12]).

Theorem 2.1 Suppose that $u_{0} \in H_{0}^{2}(I)$. Then there exists a unique global solution $u(x, t)$ for problem (8) such that

$$
u \in L^{\infty}\left(0, T ; H_{0}^{2}(I)\right) \cap L^{2}\left(0, T ; H^{4}(I)\right), \quad u_{t} \in L^{2}\left(0, T ; L^{2}(I)\right) .
$$

\section{Semi-discrete scheme}

The interval $0=x_{0}<x_{1}<\cdots<x_{L}=1$ is divided into $L$ equal finite elements by $h=1 / L$. Considering that the approximate solution must satisfy the boundary conditions, we introduce extended virtual nodes $x_{-3}, x_{-2}, x_{-1}, x_{L+1}, x_{L+2}, x_{L+3}$. If $\varphi_{i}(x)=N_{4}\left(\frac{x-x_{i}}{h}\right)$, thus $\varphi_{i}(x)$ are the cubic B-spline functions with knots at the points $x_{i}$.

Actually, B-spline basis functions are constructed as follows:

$$
\left\{\widetilde{\varphi}_{-3}(x), \widetilde{\varphi}_{-2}(x), \widetilde{\varphi}_{-1}(x), \varphi_{0}(x), \ldots, \varphi_{L-4}(x), \widetilde{\varphi}_{L-3}(x), \widetilde{\varphi}_{L-2}(x), \widetilde{\varphi}_{L-1}(x)\right\}
$$

where

$$
\begin{aligned}
& \widetilde{\varphi}_{-3}(x)=6 \varphi_{-3}(x), \\
& \widetilde{\varphi}_{-2}(x)=\varphi_{-2}(x)-4 \varphi_{-3}(x), \\
& \widetilde{\varphi}_{-1}(x)=\varphi_{-1}(x)-\frac{1}{2} \varphi_{-2}(x)+\varphi_{-3}(x), \\
& \widetilde{\varphi}_{L-3}(x)=\varphi_{L-3}(x)-\frac{1}{2} \varphi_{L-2}(x)+\varphi_{L-1}(x), \\
& \widetilde{\varphi}_{L-2}(x)=\varphi_{L-2}(x)-4 \varphi_{L-1}(x), \\
& \widetilde{\varphi}_{L-1}(x)=6 \varphi_{L-1}(x) .
\end{aligned}
$$

For convenience, we still denote the basis functions as $\left\{\varphi_{i}(x)\right\}$. The basis functions satisfy the following properties:

$$
\begin{array}{lll}
\varphi_{-3}(0)=1, & \varphi_{i}(0)=0 \quad(i \neq-3), & \varphi_{i}^{\prime}(0)=0 \quad(i \neq-3,-2), \\
\varphi_{L-1}(1)=1, & \varphi_{i}(1)=0 \quad(i \neq L-1), & \varphi_{i}^{\prime}(1)=0 \quad(i \neq L-1, L-2) .
\end{array}
$$

The B-spline parametrization is generally defined in the domain $[0,1]$. In this paper, we use $N_{4}(x)$ to define the shape functions, which is convenient in computing integrals.

Let $U_{h}$ be the cubic B-spline space, thus $U_{h} \subset H_{0}^{2}(I)$. The approximation solution $u_{h}(x, t) \in U_{h}$ satisfies

$$
u_{h}(x, t)=\sum_{i=-1}^{L-3} \delta_{i}(t) \varphi_{i}(x)
$$

where $\delta_{i}(t)$ are time-dependent quantities. 
The semi-discrete finite element scheme based on B-splines for problem (8) is: Find $u_{h}=u_{h}(\cdot, t) \in U_{h}(0<t \leq T)$ such that

$$
\left\{\begin{array}{l}
\left(u_{h, t}, v_{h}\right)+\left(\alpha(x, t) D^{2} u_{h}, D^{2} v_{h}\right)=\mu\left(\frac{D u_{h}}{1+\left|D u_{h}\right|^{2}}, D v_{h}\right), \quad \forall v_{h} \in U_{h}, \\
\left(u_{h}(0)-u_{0}, v_{h}\right)=0, \quad \forall v_{h} \in U_{h} .
\end{array}\right.
$$

In order to deduce the error estimates of the B-spline FEM, we introduce the following lemma.

Lemma 3.1 The elliptic projection $R_{h}: u \rightarrow R_{h} u \in U_{h}$ is defined by

$$
a\left(u-R_{h} u, v_{h}\right) \equiv\left(\alpha(x, t) D^{2}\left(u-R_{h} u\right), D^{2} v_{h}\right)=0, \quad \forall v_{h} \in U_{h} .
$$

It then follows from (6) and (10) that

$$
a(u, u)=a(\alpha(x, t) u, u) \geq C\|u\|_{2}^{2}, \quad \forall u \in H_{0}^{2}(I),
$$

where $C$ is a positive constant depending on $\alpha(x, t)$. Hence, $a(u, v)$ is a symmetrical positive definite bilinear form, and (see [13])

$$
\left\|u-R_{h} u\right\|+h\left\|u-R_{h} u\right\|_{1}+h^{2}\left\|u-R_{h} u\right\|_{2} \leq C h^{4}\|u\|_{4} .
$$

Firstly, we analyze the boundedness of the semi-discrete scheme.

Theorem 3.1 For $u_{h}(0) \in H_{0}^{2}(I)$, there exists a unique solution $u_{h}(t) \in U_{h}$ for problem (8) such that

$$
\left\|u_{h}(t)\right\|_{2} \leq C\left\|u_{h}(0)\right\|_{2}, \quad 0 \leq t \leq T
$$

where $C$ is a positive constant depending on $\alpha(x, t), \mu$, and $T$, but independent of mesh size $h$.

Proof According to ordinary differential equation theory, there exists a unique local solution to problem (9) in the interval $\left[0, t_{n}\right)$. If we have (13), then according to the extension theorem, we can obtain the existence of unique global solution. So, we only need to prove (13).

Setting $v_{h}=u_{h}$ in (9) and based on (6), we get

$$
\begin{aligned}
\frac{1}{2} \frac{d}{d t}\left\|u_{h}\right\|^{2}+s\left\|D^{2} u_{h}\right\|^{2} & \leq \mu\left(\frac{D u_{h}}{1+\left|D u_{h}\right|^{2}}, D u_{h}\right) \leq \mu\left\|D u_{h}\right\|^{2} \\
& =-\mu\left(D^{2} u_{h}, u_{h}\right) \leq \frac{s}{2}\left\|D^{2} u_{h}\right\|^{2}+\frac{\mu^{2}}{2 s}\left\|u_{h}\right\|^{2} .
\end{aligned}
$$

Therefore

$$
\frac{d}{d t}\left\|u_{h}\right\|^{2}+s\left\|D^{2} u_{h}\right\|^{2} \leq \frac{\mu^{2}}{s}\left\|u_{h}\right\|^{2} .
$$


Then

$$
e^{-\frac{\mu^{2}}{s} t} \frac{d}{d t}\left\|u_{h}\right\|^{2}-\frac{\mu^{2}}{s} e^{-\frac{\mu^{2}}{s} t} \frac{\mu^{2}}{s}\left\|u_{h}\right\|^{2} \leq 0
$$

We obtain

$$
\frac{d}{d t}\left(e^{-\frac{\mu^{2}}{s} t}\left\|u_{h}\right\|^{2}\right) \leq 0
$$

Integrating (15) with respect to $t$, we have

$$
\left\|u_{h}(t)\right\|^{2} \leq e^{\frac{\mu^{2}}{s} t}\left\|u_{h}(0)\right\|^{2} \leq e^{\frac{\mu^{2}}{s} T}\left\|u_{h}(0)\right\|^{2}, \quad 0 \leq t \leq T .
$$

Letting $v_{h}=u_{h, t}$ in (9), one can get

$$
\left\|u_{h, t}\right\|^{2}+\left(\alpha(x, t) D^{2} u_{h}, D^{2} u_{h, t}\right)=\mu\left(\frac{D u_{h}}{1+\left|D u_{h}\right|^{2}}, D u_{h, t}\right) .
$$

A direct calculation gives

$$
\left(\alpha(x, t) D^{2} u_{h}, D^{2} u_{h, t}\right)=\frac{1}{2} \frac{d}{d t}\left(\alpha(x, t) D^{2} u_{h}, D^{2} u_{h}\right)-\frac{1}{2}\left(\frac{\partial \alpha}{\partial t} D^{2} u_{h}, D^{2} u_{h}\right)
$$

By $\varepsilon$-inequality, we have

$$
\begin{aligned}
\mu\left(\frac{D u_{h}}{1+\left|D u_{h}\right|^{2}}, D u_{h, t}\right) & =-\mu\left(\frac{1-\left|D u_{h}\right|^{2}}{\left(1+\left|D u_{h}\right|^{2}\right)^{2}} D^{2} u_{h}, u_{h, t}\right) \\
& \leq \mu\left|\frac{1-\left|D u_{h}\right|^{2}}{\left(1+\left|D u_{h}\right|^{2}\right)^{2}}\right|_{\infty}\left\|D^{2} u_{h}\right\|\left\|u_{h, t}\right\| \leq \frac{5 \mu}{4}\left\|D^{2} u_{h}\right\|\left\|u_{h, t}\right\| \\
& \leq \frac{1}{2}\left\|u_{h, t}\right\|^{2}+\frac{25 \mu^{2}}{32}\left\|D^{2} u_{h}\right\|^{2} .
\end{aligned}
$$

It is easy to see that

$$
\left\|u_{h, t}\right\|^{2}+s \frac{d}{d t}\left\|D^{2} u_{h}\right\|^{2} \leq\left(M+\frac{25 \mu^{2}}{16}\right)\left\|D^{2} u_{h}\right\|^{2}
$$

Integrating (18) with respect to $t$, we obtain

$$
\left\|D^{2} u_{h}(t)\right\|^{2} \leq e^{\frac{16 M+25 \mu^{2}}{16 s} T}\left\|D^{2} u_{h}(0)\right\|^{2}, \quad 0 \leq t \leq T .
$$

Notice that

$$
\left\|D u_{h}\right\|^{2}=-\left(D^{2} u_{h}, u_{h}\right) \leq \frac{1}{2}\left\|D^{2} u_{h}\right\|^{2}+\frac{1}{2}\left\|u_{h}\right\|^{2},
$$

which gives (13).

Now, we give the error estimates of the finite element scheme (9). 
Theorem 3.2 Let $u$ be the solution to (8), $u_{h}$ be the solution to (9). Assume that $u(0) \in$ $H^{4}(I), u_{t} \in L^{2}\left(0, T ; H^{4}(I)\right)$, and the initial value satisfies

$$
\left\|u(0)-u_{h}(0)\right\| \leq C h^{4}\|u(0)\|_{4}
$$

Then, for $0<t \leq T$, we have the following error estimate:

$$
\left\|u(t)-u_{h}(t)\right\| \leq C h^{3}\left[h\|u(0)\|_{4}+\left(\int_{0}^{t}\left(\|u(\tau)\|_{4}^{2}+h^{2}\left\|u_{t}(\tau)\right\|_{4}^{2}\right) d \tau\right)^{\frac{1}{2}}\right] .
$$

Proof Let $\theta(t)=R_{h} u-u_{h}, \rho(t)=u-R_{h} u$, then we have

$$
u-u_{h}=u-R_{h} u+R_{h} u-u_{h}=\theta(t)+\rho(t) .
$$

It follows from (8)-(9) that

$$
\begin{aligned}
& \left(\theta_{t}, v_{h}\right)+\left(\alpha(x, t) D^{2} \theta, D^{2} v_{h}\right) \\
& \quad=-\left(\rho_{t}, v_{h}\right)+\mu\left(\frac{D u}{1+|D u|^{2}}-\frac{D u_{h}}{1+\left|D u_{h}\right|^{2}}, D v_{h}\right)
\end{aligned}
$$

Setting $v_{h}=\theta$ in (22), we have

$$
\left(\theta_{t}, \theta\right)+\left(\alpha(x, t) D^{2} \theta, D^{2} \theta\right)=-\left(\rho_{t}, \theta\right)+\mu\left(\frac{D u}{1+|D u|^{2}}-\frac{D u_{h}}{1+\left|D u_{h}\right|^{2}}, D \theta\right) .
$$

Using (6) and $\varepsilon$-inequality, we can deduce that

$$
\begin{aligned}
& \frac{1}{2} \frac{d}{d t}\|\theta\|^{2}+s\left\|D^{2} \theta\right\|^{2} \\
& \quad \leq\left|-\left(\rho_{t}, \theta\right)\right|+\mu\left\|\frac{D u}{1+|D u|^{2}}-\frac{D u_{h}}{1+\left|D u_{h}\right|^{2}}\right\|\|D \theta\| \\
& \quad \leq \frac{1}{2}\left(\left\|\rho_{t}\right\|^{2}+\|\theta\|^{2}\right)+\mu\left\|\frac{D u}{1+|D u|^{2}}-\frac{D u_{h}}{1+\left|D u_{h}\right|^{2}}\right\|^{2}+\frac{\mu}{4}\|D \theta\|^{2} .
\end{aligned}
$$

Note that

$$
\begin{aligned}
\left\|\frac{D u}{1+|D u|^{2}}-\frac{D u_{h}}{1+\left|D u_{h}\right|^{2}}\right\|^{2} & \leq\left\|\frac{1-D u D u_{h}}{\left(1+|D u|^{2}\right)\left(1+\left|D u_{h}\right|^{2}\right)}\left(D u-D u_{h}\right)\right\|^{2} \\
& \leq\left|\frac{1-D u D u_{h}}{\left(1+|D u|^{2}\right)\left(1+\left|D u_{h}\right|^{2}\right)}\right|_{\infty}^{2}\left\|D u-D u_{h}\right\|^{2} \\
& \leq \frac{25}{8}\left(\|D \theta\|^{2}+\|D \rho\|^{2}\right) .
\end{aligned}
$$


Hence

$$
\begin{aligned}
\frac{1}{2} & \frac{d}{d t}\|\theta\|^{2}+s\left\|D^{2} \theta\right\|^{2} \\
& \leq \frac{1}{2}\left\|\rho_{t}\right\|^{2}+\frac{25 \mu}{8}\|D \rho\|^{2}+\frac{1}{2}\|\theta\|^{2}+\frac{27 \mu}{8}\|D \theta\|^{2} \\
& \leq \frac{1}{2}\left\|\rho_{t}\right\|^{2}+\frac{25 \mu}{8}\|D \rho\|^{2}+\frac{s}{2}\left\|D^{2} \theta\right\|^{2}+\left(\frac{1}{2}+\frac{729 \mu^{2}}{128 s}\right)\|\theta\|^{2} .
\end{aligned}
$$

Therefore we have

$$
\frac{d}{d t}\|\theta\|^{2}+s\left\|D^{2} \theta\right\|^{2} \leq\left\|\rho_{t}\right\|^{2}+\frac{25 \mu}{4}\|D \rho\|^{2}+\left(1+\frac{729 \mu^{2}}{64 s}\right)\|\theta\|^{2} .
$$

By Gronwall's inequality, we get

$$
\|\theta\|^{2} \leq C\left[\|\theta(0)\|^{2}+\int_{0}^{t}\left(\left\|\rho_{t}\right\|^{2}+\|D \rho\|^{2}\right) d \tau\right]
$$

Using (12) and (20), it is easy to see that

$$
\begin{aligned}
\|\theta(0)\| & =\left\|u(0)-u_{h}(0)+R_{h} u(0)-u(0)\right\| \leq\left\|u(0)-u_{h}(0)\right\|+\|\rho(0)\| \\
& \leq\left\|u(0)-u_{h}(0)\right\|+C h^{4}\|u(0)\|_{4} \leq C^{\prime} h^{4}\|u(0)\|_{4},
\end{aligned}
$$

and

$$
\begin{aligned}
& \|D \rho\|=\left\|D\left(u_{h}-R_{h} u\right)\right\| \leq C h^{3}\|u\|_{4}, \\
& \left\|\rho_{t}\right\|=\left\|u_{t}-R_{h} u_{t}\right\| \leq C h^{4}\left\|u_{t}\right\|_{4} .
\end{aligned}
$$

Finally, for $0<t \leq T$, it follows from (28)-(30) that the proof of the theorem is completed.

Next, we deal with the $H^{2}$-estimate.

Theorem 3.3 Let $u$ be the solution to (8), $u_{h}$ be the solution to (9). Assume that $u(0) \in$ $H^{4}(I), u, u_{t} \in L^{2}\left(0, T ; H^{4}(I)\right)$, and the initial value satisfies

$$
\left\|u(0)-u_{h}(0)\right\|_{2} \leq C h^{2}\|u(0)\|_{4} .
$$

Then we have the following error estimate:

$$
\left\|u(t)-u_{h}(t)\right\|_{2} \leq C h^{2}\left[\|u(0)\|_{4}+\left(\int_{0}^{t}\left(\|u(\tau)\|_{4}^{2}+h^{4}\left\|u_{t}(\tau)\right\|_{4}^{2}\right) d \tau\right)^{\frac{1}{2}}\right]
$$

Proof Letting $v_{h}=\theta_{t}$ in (22), we get

$$
\left\|\theta_{t}\right\|^{2}+\left(\alpha(x, t) D^{2} \theta, D^{2} \theta_{t}\right)=-\left(\rho_{t}, \theta_{t}\right)+\mu\left(\frac{D u}{1+|D u|^{2}}-\frac{D u_{h}}{1+\left|D u_{h}\right|^{2}}, D \theta_{t}\right),
$$


where

$$
\left(\alpha(x, t) D^{2} \theta, D^{2} \theta_{t}\right)=\frac{1}{2} \frac{d}{d t}\left(\alpha(x, t) D^{2} \theta, D^{2} \theta\right)-\frac{1}{2}\left(\frac{\partial \alpha}{\partial t} D^{2} \theta, D^{2} \theta\right) .
$$

Based on (6) and $\varepsilon$-inequality, we have

$$
\begin{aligned}
\left\|\theta_{t}\right\|^{2}+\frac{s}{2} \frac{d}{d t}\left\|D^{2} \theta\right\|^{2} \leq & \frac{M}{2}\left\|D^{2} \theta\right\|^{2}+\left\|\rho_{t}\right\|^{2}+\frac{1}{4}\left\|\theta_{t}\right\|^{2}+\frac{1}{4}\left\|\theta_{t}\right\|^{2} \\
& +\mu^{2}\left\|D\left(\frac{D u}{1+|D u|^{2}}-\frac{D u_{h}}{1+\left|D u_{h}\right|^{2}}\right)\right\|^{2} .
\end{aligned}
$$

To derive the theorem, we give the following estimate:

$$
\begin{aligned}
\| D & \left(\frac{D u}{1+|D u|^{2}}-\frac{D u_{h}}{1+\left|D u_{h}\right|^{2}}\right) \|^{2} \\
= & \left\|\frac{\left(1-|D u|^{2}\right) D^{2} u}{\left(1+|D u|^{2}\right)^{2}}-\frac{\left(1-\left|D u_{h}\right|^{2}\right) D^{2} u_{h}}{\left(1+\left|D u_{h}\right|^{2}\right)^{2}}\right\|^{2} \\
\leq & 2\left\|\left(\frac{1-|D u|^{2}}{\left(1+|D u|^{2}\right)^{2}}-\frac{1-\left|D u_{h}\right|^{2}}{\left(1+\left|D u_{h}\right|^{2}\right)^{2}}\right) D^{2} u\right\|^{2} \\
& +2\left\|\frac{1-\left|D u_{h}\right|^{2}}{\left(1+\left|D u_{h}\right|^{2}\right)^{2}}\left(D^{2} \theta+D^{2} \rho\right)\right\|^{2} \\
\leq & 2\left|\frac{\left(D u+D u_{h}\right)\left(-3-|D u|^{2}-\left|D u_{h}\right|^{2}+|D u|^{2}\left|D u_{h}\right|^{2}\right)}{\left(1+|D u|^{2}\right)^{2}\left(1+\left|D u_{h}\right|^{2}\right)^{2}}\right|_{\infty}^{2}\left\|D^{2} u\right\|^{2}\|D \theta+D \rho\|^{2} \\
& +2\left|\frac{1-\left|D u_{h}\right|^{2}}{\left(1+\left|D u_{h}\right|^{2}\right)^{2}}\right|_{\infty}^{2}\left\|D^{2} \theta+D^{2} \rho\right\|^{2} \\
\leq & C\left(\left\|D^{2} \theta\right\|^{2}+\left\|D^{2} \rho\right\|^{2}+\|D \theta\|^{2}+\|D \rho\|^{2}\right) \\
\leq & C^{\prime}\left(\left\|D^{2} \theta\right\|^{2}+\|\theta\|^{2}+\|\rho\|_{2}^{2}\right) .
\end{aligned}
$$

Hence

$$
\left\|\theta_{t}\right\|^{2}+s \frac{d}{d t}\left\|D^{2} \theta\right\|^{2} \leq C\left(\left\|D^{2} \theta\right\|^{2}+\|\theta\|^{2}\|+\| \rho_{t}\left\|^{2}+\right\| \rho \|_{2}^{2}\right) .
$$

Integrating (36) with respect to $t$, we have

$$
\left\|D^{2} \theta\right\|^{2} \leq C\left(\left\|D^{2} \theta(0)\right\|^{2}+\int_{0}^{t}\left(\|\theta\|^{2}+\left\|\rho_{t}\right\|^{2}+\|\rho\|_{2}^{2}\right) d \tau\right) .
$$

Combining (12) and (31), we have

$$
\begin{aligned}
\left\|D^{2} \theta(0)\right\| & \leq\left\|D^{2} u(0)-D^{2} u_{h}(0)\right\|+\left\|D^{2} R_{h} u(0)-D^{2} u(0)\right\| \\
& \leq C h^{2}\|u(0)\|_{4} .
\end{aligned}
$$

Then we obtain

$$
\left\|D^{2} \theta\right\| \leq C h^{2}\left\{\|u(0)\|_{4}+\left[\int_{0}^{t}\left(\|u(\tau)\|_{4}^{2}+h^{4}\left\|u_{t}(\tau)\right\|_{4}^{2}\right) d \tau\right]^{\frac{1}{2}}\right\} .
$$

Using the triangle inequality, we complete the proof. 


\section{Fully-discrete scheme}

The fully-discrete finite element scheme for problem (8) is: Find $u_{h}^{n} \in U_{h}(n=1,2, \ldots, N)$ such that

$$
\left\{\begin{array}{l}
\left(\partial_{t} u_{h}^{n}, v_{h}\right)+\left(\alpha\left(x, t^{n, \theta}\right) D^{2} u_{h}^{n, \theta}, D^{2} v_{h}\right)=\mu\left(\frac{D u_{h}^{n, \theta}}{1+\left|D u_{h}^{n, \vartheta}\right|^{2}}, D v_{h}\right), \quad \forall v_{h} \in U_{h}, \\
\left(u(0), v_{h}\right)=\left(u_{h}^{0}, v_{h}\right), \quad \forall v_{h} \in U_{h},
\end{array}\right.
$$

where $N$ is a given positive integer, $\Delta t=T / N$ denotes the time step size, $t_{n}=n \Delta t$ and

$$
\begin{aligned}
& \partial_{t} u_{h}^{n}=\left(u_{h}^{n}-u_{h}^{n-1}\right) / \Delta t, \\
& t^{n, \theta}=\theta t^{n}+(1-\theta) t^{n-1}, \\
& u_{h}^{n, \theta}=\theta u_{h}^{n}+(1-\theta) u_{h}^{n-1}, \\
& u_{h}^{n, \vartheta}=\vartheta u_{h}^{n}+(1-\vartheta) u_{h}^{n-1} .
\end{aligned}
$$

When $\theta=\vartheta=1$ and $\theta=\vartheta=\frac{1}{2}$, the schemes are the backward Euler and Crank-Nicolson finite element scheme, respectively.

In this paper, we choose $\theta=1, \vartheta=0$. The fully-discrete finite element scheme is

$$
\left\{\begin{array}{l}
\left(\partial_{t} u_{h}^{n}, v_{h}\right)+\left(\alpha\left(x, t^{n}\right) D^{2} u_{h}^{n}, D^{2} v_{h}\right)=\mu\left(\frac{D u_{h}^{n}}{1+\left|D u_{h}^{h-1}\right|^{2}}, D v_{h}\right), \quad \forall v_{h} \in U_{h}, \\
\left(u(0)-u_{h}^{0}, v_{h}\right)=0, \quad \forall v_{h} \in U_{h} .
\end{array}\right.
$$

From the above formulation, if $u_{h}^{n-1}$ is known, the fully-discrete finite element scheme is linear. We give the next estimate.

Theorem 4.1 Let $u^{n}$ be the solution to problem (8), $u_{h}^{n}$ be the solution to the fully-discrete scheme (40). Assume that $u(0) \in H^{4}(I), u_{t} \in L^{2}\left(0, T ; H^{4}(I)\right), u_{t t} \in L^{2}\left(0, T ; L^{2}(I)\right)$, and $u_{0 h} \in$ $U_{h}$ satisfies

$$
\left\|u(0)-u_{h}^{0}\right\| \leq C h^{4}\|u(0)\|_{4}
$$

For sufficiently small $\Delta t$, there exists a constant $C$ independent of $h, \Delta t$, and $n$ such that

$$
\left\|u^{n}-u_{h}^{n}\right\| \leq C\left(\Delta t+h^{3}\right) .
$$

Proof Let $\rho^{n}=u^{n}-R_{h} u^{n}$ and $\theta^{n}=R_{h} u^{n}-u_{h}^{n}$. Then $u^{n}-u_{h}^{n}=\rho^{n}+\theta^{n}$, and

$$
\begin{aligned}
& \left(\partial_{t} \theta^{n}, v_{h}\right)+\left(\alpha^{n} D^{2} \theta^{n}, D^{2} v_{h}\right) \\
& \quad=\left(r^{n}, v_{h}\right)+\mu\left(\frac{D u^{n}}{1+\left|D u^{n}\right|^{2}}-\frac{D u_{h}^{n}}{1+\left|D u_{h}^{n-1}\right|^{2}}, D v_{h}\right),
\end{aligned}
$$

where

$$
\partial_{t} \theta^{n}=\left(\theta^{n}-\theta^{n-1}\right) / \Delta t, \quad \alpha^{n}=\alpha\left(x, t^{n}\right), \quad r^{n}=\partial_{t} R_{h} u\left(t_{n}\right)-u_{t}\left(t_{n}\right) .
$$


Taking $v_{h}=\theta^{n}$ in (43), using Lemma 3.1 and $\varepsilon$-inequality, we get

$$
\begin{aligned}
\left\|\theta^{n}\right\|^{2}+s \Delta t\left\|D^{2} \theta^{n}\right\|^{2} \leq & \frac{1}{2}\left(\left\|\theta^{n-1}\right\|^{2}+\left\|\theta^{n}\right\|^{2}\right)+\frac{\Delta t}{2}\left(\left\|r^{n}\right\|^{2}+\left\|\theta^{n}\right\|^{2}\right) \\
& +\Delta t\left\|D \theta^{n}\right\|^{2}+\frac{\mu^{2} \Delta t}{4}\left\|\frac{D u^{n}}{1+\left|D u^{n}\right|^{2}}-\frac{D u_{h}^{n}}{1+\left|D u_{h}^{n-1}\right|^{2}}\right\|^{2} .
\end{aligned}
$$

We can easily obtain

$$
\left\|D \theta^{n}\right\|^{2}=-\left(\theta^{n}, D^{2} \theta^{n}\right) \leq \frac{1}{2 s}\left\|\theta^{n}\right\|^{2}+\frac{s}{2}\left\|D^{2} \theta^{n}\right\|^{2}
$$

Therefore

$$
\begin{aligned}
\left\|\theta^{n}\right\|^{2}+s \Delta t\left\|D^{2} \theta^{n}\right\|^{2} \leq & \left\|\theta^{n-1}\right\|^{2}+\Delta t\left(1+\frac{1}{s}\right)\left\|\theta^{n}\right\|^{2}+\Delta t\left\|r^{n}\right\|^{2} \\
& +\frac{\mu^{2} \Delta t}{2}\left\|\frac{D u^{n}}{1+\left|D u^{n}\right|^{2}}-\frac{D u_{h}^{n}}{1+\left|D u_{h}^{n-1}\right|^{2}}\right\|^{2}
\end{aligned}
$$

In addition, we have

$$
\begin{aligned}
& \left\|\frac{D u^{n}}{1+\left|D u^{n}\right|^{2}}-\frac{D u_{h}^{n}}{1+\left|D u_{h}^{n-1}\right|^{2}}\right\| \\
& =\left\|\frac{D u^{n}}{1+\left|D u^{n}\right|^{2}}-\frac{D u^{n}}{1+\left|D u^{n-1}\right|^{2}}\right\|+\left\|\frac{D u^{n}}{1+\left|D u^{n-1}\right|^{2}}-\frac{D u^{n}}{1+\left|D u_{h}^{n-1}\right|^{2}}\right\| \\
& +\left\|\frac{D u^{n}}{1+\left|D u_{h}^{n-1}\right|^{2}}-\frac{D u_{h}^{n}}{1+\left|D u_{h}^{n-1}\right|^{2}}\right\| \\
& \leq\left\|\frac{D u^{n}\left(D u^{n}+D u^{n-1}\right)\left(D u^{n}-D u^{n-1}\right)}{\left(1+\left|D u^{n}\right|^{2}\right)\left(1+\left|D u^{n-1}\right|^{2}\right)}\right\| \\
& +\left\|\frac{D u^{n}\left(D u^{n-1}+D u_{h}^{n-1}\right)\left(D u^{n-1}-D u_{h}^{n-1}\right)}{\left(1+\left|D u^{n-1}\right|^{2}\right)\left(1+\left|D u_{h}^{n-1}\right|^{2}\right)}\right\|+\left\|\frac{D u^{n}-D u_{h}^{n}}{1+\left|D u_{h}^{n-1}\right|^{2}}\right\| \\
& \leq\left|D u^{n}\right|_{\infty}\left\|D u^{n}-D u^{n-1}\right\|+\left|D u^{n}\right|_{\infty}\left\|D u^{n-1}-D u_{h}^{n-1}\right\| \\
& +\left\|D u^{n}-D u_{h}^{n}\right\| \text {. }
\end{aligned}
$$

By Sobolev's embedding theorem, we know $W^{2,0}(I) \hookrightarrow W^{1, \infty}(I)$, that is,

$$
\left|D u^{n}\right|_{\infty} \leq\left\|u^{n}\right\|_{2} \leq C
$$

Note that

$$
\left|D u^{n}-D u^{n-1}\right|=\left|\int_{t_{n-1}}^{t_{n}} D u_{t}(t) d t\right| \leq \Delta t^{\frac{1}{2}}\left(\int_{t_{n-1}}^{t_{n}}\left\|D u_{t}(t)\right\|^{2} d t\right)^{\frac{1}{2}} .
$$

Adding (46)-(48) together, we have

$$
\left\|\frac{D u^{n}}{1+\left|D u^{n}\right|^{2}}-\frac{D u_{h}^{n}}{1+\left|D u_{h}^{n-1}\right|^{2}}\right\|
$$




$$
\begin{aligned}
& \leq C\left[\Delta t^{\frac{1}{2}}\left(\int_{t_{n-1}}^{t_{n}}\left\|D u_{t}(t)\right\|^{2} d t\right)^{\frac{1}{2}}+\left\|D \rho^{n-1}+D \theta^{n-1}\right\|+\left\|D \rho^{n}+D \theta^{n}\right\|\right] \\
& \leq C\left[\Delta t^{\frac{1}{2}}\left(\int_{t_{n-1}}^{t_{n}}\left\|D u_{t}(t)\right\|^{2} d t\right)^{\frac{1}{2}}+h^{k-1}+\left\|D \theta^{n-1}\right\|+\left\|D \theta^{n}\right\|\right] .
\end{aligned}
$$

Let $r^{n}=r_{1}^{n}+r_{2}^{n}$, where

$$
\begin{aligned}
& r_{1}^{j}=\partial_{t} R_{h} u\left(t_{j}\right)-\partial_{t} u\left(t_{j}\right)=\frac{1}{\Delta t} \int_{t_{j-1}}^{t_{j}}\left(R_{h}-I\right) u_{t} d t, \\
& r_{2}^{j}=\partial_{t} u\left(t_{j}\right)-u_{t}\left(t_{j}\right)=-\frac{1}{\Delta t} \int_{t_{j-1}}^{t_{j}}\left(t-t_{j-1}\right) u_{t t} d t .
\end{aligned}
$$

It is easy to see that

$$
\left\|r_{1}^{j}\right\| \leq \frac{1}{\Delta t} \int_{t_{j-1}}^{t_{j}} C h^{4}\left\|u_{t}\right\|_{k} d t \leq C(\Delta t)^{-\frac{1}{2}} h^{4}\left(\int_{t_{j-1}}^{t_{j}}\left\|u_{t}\right\|_{4}^{2} d t\right)^{\frac{1}{2}}
$$

and

$$
\left\|r_{2}^{j}\right\| \leq \int_{t_{j-1}}^{t_{j}}\left\|u_{t t}\right\| d t \leq(\Delta t)^{\frac{1}{2}}\left(\int_{t_{j-1}}^{t_{j}}\left\|u_{t t}\right\|^{2} d t\right)^{\frac{1}{2}} .
$$

One can easily get

$$
\sum_{j=1}^{n}\left\|r^{j}\right\|^{2} \leq C(\Delta t)^{-1}\left[(\Delta t)^{2}+h^{8}\right] \int_{0}^{t_{n}}\left(\left\|u_{t}\right\|_{4}^{2}+\left\|u_{t t}\right\|^{2}\right) d t .
$$

Substituting (49) and (50) into (45), we obtain

$$
\begin{aligned}
& \left\|\theta^{n}\right\|^{2}-\left\|\theta^{n-1}\right\|^{2}+\frac{s \Delta t}{2}\left(\left\|D^{2} \theta^{n}\right\|^{2}-\left\|D^{2} \theta^{n-1}\right\|^{2}\right) \\
& \quad \leq C \Delta t\left(\left\|\theta^{n-1}\right\|^{2}+\left\|\theta^{n}\right\|^{2}+h^{6}\right)+C\left[(\Delta t)^{2}+h^{8}\right] \int_{t_{n-1}}^{t_{n}}\left(\left\|u_{t}\right\|_{4}^{2}+\left\|u_{t t}\right\|^{2}\right) d t .
\end{aligned}
$$

Taking the sum over $n$, by $n \Delta t=t_{n} \leq T$, we have

$$
\begin{aligned}
& \left\|\theta^{n}\right\|^{2}+\frac{s \Delta t}{2}\left\|D^{2} \theta^{n}\right\|^{2} \\
& \leq(1+C \Delta t)\left\|\theta^{0}\right\|^{2}+\frac{s \Delta t}{2}\left\|D^{2} \theta^{0}\right\|^{2}+C\left(\Delta t \sum_{i=1}^{n}\left\|\theta^{i}\right\|^{2}+T h^{6}\right) \\
& \quad+C\left[(\Delta t)^{2}+h^{8}\right] \int_{t_{0}}^{t_{n}}\left(\left\|u_{t}\right\|_{4}^{2}+\left\|u_{t t}\right\|^{2}\right) d t .
\end{aligned}
$$

Using (12) and (41), we get

$$
\left\|\theta^{0}\right\| \leq\left\|u(0)-u_{h}(0)\right\|+\left\|u(0)-R_{h} u(0)\right\| \leq C h^{4}\|u(0)\|_{4} .
$$


Thus

$$
\left\|\theta^{n}\right\|^{2} \leq C \Delta t \sum_{i=1}^{n-1}\left\|\theta^{i}\right\|^{2}+C\left[(\Delta t)^{2}+h^{6}\right] .
$$

Using the discrete Gronwall inequality and Lemma 3.1, we get the error estimate (42).

Although we assume that $\alpha(x, t)$ is a smooth function in $(5), \alpha(x, t) \in W^{1, \infty}\left(0, T ; W^{1, \infty}(I)\right)$ is enough.

It is difficult to estimate the nonlinear term which affects the convergence order of the schemes. It leads to the failure of the optimal convergence.

\section{Numerical approximation}

In this section, we consider the following equation:

$$
\left\{\begin{array}{l}
u_{t}+\left(\alpha(x, t) u_{x x}\right)_{x x}+\left(\frac{u_{x}}{1+\left|u_{x}\right|^{2}}\right)_{x}=f(x, t), \quad(x, t) \in(0,1) \times(0,1] \\
u(x, t)=u_{x}(x, t)=0, \quad x=0,1, t \in(0,1] \\
u(x, 0)=u_{0}(x), \quad x \in[0,1]
\end{array}\right.
$$

where $\alpha(x, t)=1+x t$. We take the analytical solution $u(x, t)=t^{2}(1-\cos 2 \pi x)$. The behavior of the exact solution to equation (53) is shown in Fig. 1 and the profile of the solution to the fully-discrete form is given in Fig. 2.

The numerical solution is in good accordance with the exact solution, indicating that the numerical scheme is valid and efficient.

Then the errors and the orders of convergence are shown in Tables 1-3.

\subsection{Tables}

In Table 1 , taking the time step $\Delta t=\frac{1}{200,000}$, we find that the rate of convergence in space is the fourth order in $L^{2}$ norm and is the second order in $H^{2}$ norm. Tt means that the accuracy in space is better than the theoretical precision.

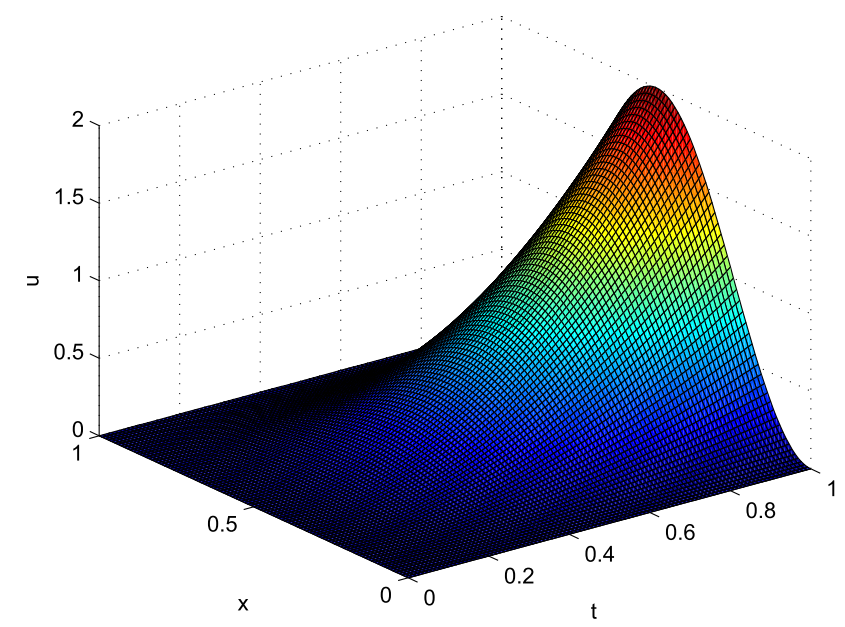

Figure 1 The exact solution to the full-discrete scheme. The figure is the profile of the exact solution to the cubic B-spline finite element method 


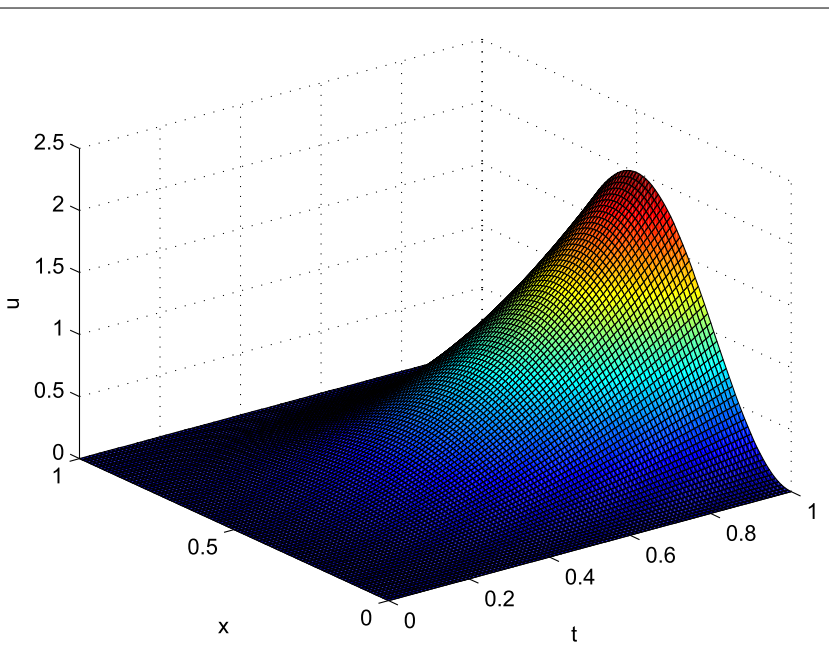

Figure 2 The numerical solution to the full-discrete scheme. The figure is the profile of the numerical solution to the cubic B-spline finite element method

Table 1 The error for different space step $h$ at $t=1$

\begin{tabular}{lllllll}
\hline$(\Delta t, h)$ & $\left\|u-u_{h}\right\|$ & Rate & $\left\|u-u_{h}\right\|_{1}$ & Rate & $\left\|u-u_{h}\right\|_{2}$ & Rate \\
\hline$(1 / 200,000,1 / 10)$ & $2.6858 e^{-4}$ & & $7.1462 e^{-3}$ & & $4.3020 e^{-1}$ & \\
$(1 / 200,000,1 / 20)$ & $1.2314 e^{-5}$ & 4.4470 & $8.1328 e^{-4}$ & 3.1354 & $1.0389 e^{-1}$ & 2.0500 \\
$(1 / 200,000,1 / 40)$ & $7.4241 e^{-7}$ & 4.0519 & $9.9745 e^{-5}$ & 3.0274 & $2.5745 e^{-2}$ & 2.0127 \\
$(1 / 200,000,1 / 80)$ & $4.1905 e^{-8}$ & 4.1470 & $1.2399 e^{-5}$ & 3.0080 & $6.4221 e^{-3}$ & 2.0032 \\
\hline
\end{tabular}

Table 2 The error for different time step $\Delta t$ at $t=1$

\begin{tabular}{lllllll}
\hline$(\Delta t, h)$ & $\left\|u-u_{h}\right\|$ & Rate & $\left\|u-u_{h}\right\|_{1}$ & Rate & $\left\|u-u_{h}\right\|_{2}$ & Rate \\
\hline$(1 / 10,1 / 800)$ & $5.9048 e^{-4}$ & & $2.0970 e^{-3}$ & & $1.3459 e^{-2}$ & \\
$(1 / 20,1 / 800)$ & $2.7118 e^{-4}$ & 1.1226 & $9.6223 e^{-4}$ & 1.1239 & $6.1815 e^{-3}$ & 1.1225 \\
$(1 / 40,1 / 800)$ & $1.3035 e^{-4}$ & 1.0569 & $4.6234 e^{-4}$ & 1.0574 & $2.9718 e^{-3}$ & 1.0566 \\
$(1 / 80,1 / 800)$ & $6.4199 e^{-5}$ & 1.0218 & $2.2765 e^{-4}$ & 1.0221 & $1.4644 e^{-3}$ & 1.0210 \\
\hline
\end{tabular}

Table 3 The error for different time step $\Delta t$ and space step $h$ at $t=1$

\begin{tabular}{lllllll}
\hline$(\Delta t, h)$ & $\left\|u-u_{h}\right\|$ & Rate & $\left\|u-u_{h}\right\|_{1}$ & Rate & $\left\|u-u_{h}\right\|_{2}$ & Rate \\
\hline$(1 / 1000,1 / 10)$ & $2.6507 e^{-4}$ & & $7.1428 e^{-3}$ & & $4.3020 e^{-1}$ & \\
$(1 / 8000,1 / 20)$ & $1.2618 e^{-5}$ & 4.3928 & $8.1323 e^{-4}$ & 3.1348 & $1.0389 e^{-1}$ & 2.0500 \\
$(1 / 64,000,1 / 40)$ & $7.1987 e^{-7}$ & 4.1316 & $9.9738 e^{-5}$ & 3.0274 & $2.5745 e^{-2}$ & 2.0127 \\
$(1 / 512,000,1 / 80)$ & $4.1744 e^{-8}$ & 4.1081 & $1.2400 e^{-5}$ & 3.0078 & $6.4221 e^{-3}$ & 2.0032 \\
\hline
\end{tabular}

In Table 2, the space step is $h=\frac{1}{800}$. It is easy to see that the orders of error estimate are $O(\Delta t)$ in both $L^{2}$ and $H^{2}$ norms.

In Table 3, we choose $(\Delta t, h)=\left(\frac{1}{1000}, \frac{1}{10}\right),\left(\frac{1}{8000}, \frac{1}{20}\right),\left(\frac{1}{64,000}, \frac{1}{40}\right),\left(\frac{1}{512,000}, \frac{1}{80}\right)$, respectively. The errors are optimal order convergent in $L^{2}$ and $H^{2}$ norms.

The tables show the errors and convergence analysis in space and time. In $L^{2}$ norm, the convergence order is higher than the result of theoretical analysis. The reason is that it is difficult to deal with the estimation of the nonlinear term. The numerical example demonstrates that the cubic B-spline finite element scheme is efficient. 


\section{Conclusion}

In this paper, the model is a class of fourth-order nonlinear parabolic differential equations with variable coefficient $\alpha(x, t)$. By constructing appropriate basis functions satisfying the boundary conditions, we obtain the finite element schemes based on the cubic B-spline. We analyze the boundedness of the semi-discrete scheme and discuss the error estimates in $L^{2}$ norm and $H^{2}$ norm. We also discrete the temporal variable, and the fully-discrete scheme is taken as a linearized backward Euler scheme. The coefficient matrix of the corresponding linear system is spare, which can be solved efficiently. For the fully-discrete scheme, the rate of convergence is discussed. The numerical results indicate that our method is efficient and its computational accuracy is better than the theoretical results.

Through the above results, we know that the cubic B-splines have better smoothness than the Hermite type elements. The B-spline finite element has only one type of basis functions. It is clear that the B-spline FEM decreases the order of the coefficient matrix, but it can get the same convergence order as the Hermite FEM.

Acknowledgements

The authors would like to express their deep thanks for the referee's valuable suggestions for the revision and improvement of the manuscript.

Funding

This paper is supported by the Natural Science Foundation of China [grant no. 11561011].

Availability of data and materials

Not applicable.

Competing interests

The authors declare that they have no competing interests.

Authors' contributions

DQ wrote the first draft, made the figure of numerical solution and results on errors, all authors read and approved the final manuscript.

\section{Author details}

${ }^{1}$ College of Mathematics, Jilin University, Changchun, P.R. China. ${ }^{2}$ Fundamental Department, Aviation University of Air Force, Changchun, P.R. China. ${ }^{3}$ School of Biology and Engineering, Guizhou Medical University, Guiyang, P.R. China.

\section{Publisher's Note}

Springer Nature remains neutral with regard to jurisdictional claims in published maps and institutional affiliations.

Received: 26 July 2018 Accepted: 19 February 2019 Published online: 08 May 2019

References

1. Hunt, A.W., Orme, C., Williams, D.R.M., Orr, B.G., Sander, L.M.: Instabilities in MBE growth. Europhys. Lett. 27(8), 611-616 (1994)

2. Du, Q., Nicolaides, R.A.: Numerical analysis of a continuum model of phase transition. SIAM J. Numer. Anal. 28(5), 1310-1322 (1991)

3. Rost, M., Krug, J., Smilauer, P.: Unstable epitaxy on vicinal surfaces. Surf. Sci. 369(1), 393-402 (1996)

4. Johnson, M.D., Orme, C., Hunt, A.W., Graff, D., Sudijion, J., Sauder, L.M., Orr, B.G.: Stable and unstable growth in molecular beam epitaxy. Phys. Rev. Lett. 72(1), 116-119 (1994)

5. Rost, M., Krug, J.: Coarsening of surface structures in unstable epitaxial growth. Phys. Rev. E 55(4), 3952-3957 (1997)

6. Mullins, W.W.: Flatening of nearly plane surfaces due to capillarity. J. Appl. Phys. 30, 77-87 (1959)

7. Krug, J., Plischke, M., Siegert, M.: Surface diffusion currents and the universality classes of growth. Phys. Rev. Lett. 70(21), 3271-3274 (1993)

8. Politi, P., Villain, J.: Ehrlich-Schwoebel instability in molecular-beam epitaxy—a minimal model. Phys. Rev. B 54(7), 5114-5129 (1996)

9. Krug, J., Schimschak, M.: Metastability of step flow growth in $1+1$ dimensions. J. Phys. I 5(9), 1065-1086 (1995)

10. Grasselli, M., Mola, G., Yagi, A.: On the longtime behavior of solutions to a model for epitaxial growth. Osaka J. Math. 48(4), 987-1004 (2011)

11. Pierre-Louis, O., Misbah, C., Satio, Y., Krug, J., Politi, P.: New nonlinear evolution equation for steps during molecular beam epitaxy on vicinal surfaces. Phys. Rev. Lett. 80(19), 4221-4224 (1998) 
12. Zhao, X.P., Liu, F.N., Liu, B.: Finite element method for a nonlinear differential equation describing crystal surface growth. Math. Model. Anal. 19(2), 155-168 (2014)

13. Ciarlet, P.G.: The Finite Element Method for Elliptic Problems. North-Holland, Amsterdam (1978)

14. Douglas, J., Dupont, T.: Galerkin methods for parabolic equations. SIAM J. Numer. Anal. 7, 575-626 (1970)

15. Brenner, S.C., Scott, L.R.: The Mathematical Theory of Finite Element Methods. Springer, Berlin (2002)

16. Chen, $H_{\text {., }}$ Chen, Y.: A combined mixed finite element and discontinuous Galerkin method for compressible miscible displacement. SIAM J. Numer. Anal. 7, 575-626 (1970)

17. Brezzi, F., Fortin, M.: Mixed and hybrid finite element methods. Natur. Sci. J. Xiangtan Univ. 26(2), 119-126 (2004)

18. Choo, S.M., Kim, Y.H.: Finite element scheme for the viscous Cahn-Hilliard equation with a nonconstant gradient energy coefficient. J. Appl. Math. Comput. 19(1-2), 385-395 (2005)

19. Zhang, T.: Finite element analysis for Cahn-Hilliard equation. Math. Numer. Sin. 28, 281-292 (2006)

20. Elliott, C.M., French, D.A.: A nonconforming finite element method for the two-dimensional Cahn-Hilliard equation. SIAM J. Numer. Anal. 26(4), 889-903 (1989)

21. Barrett, J.W., Blowey, J.F., Garcke, H.: Finite element approximation of a fourth order nonlinear degenerate parabolic equation. Numer. Math. 80(4), 525-556 (1998)

22. Kästner, M., Metsch, P., de Borst, R.: Isogeometric analysis of the Cahn-Hilliard equation-a convergence study. J. Comput. Phys. 305, 360-371 (2016)

23. Schoenberg, I.J.: Contributions to the problem of approximation of equidistant data by analytic functions. Q. Appl. Math. 4, 45-99 (1946)

24. Curry, H.B., Schoenberg, I.J.: On Pólya frequency functions IV: the fundamental spline functions and their limits. J. Anal. Math. 17(1), 71-107 (1966)

25. de Boor, C.: B-form basis. In: Farin, G. (ed.) Geometric Modelling, pp. 131-148. SIAM, Philadelphia (1978)

26. de Boor, C., Höllig, K.: Bivariate box splines and smooth pp functions on a three directional mesh. J. Comput. Appl. Math. 9(1), 13-28 (1983)

27. Micchelli, C.A.: A constructive approach to Kergin interpolation in $R^{k}$ : multivariate $B$-splines and Lagrange interpolation. Rocky Mt. J. Math. 10(3), 485-497 (1980)

28. Dahmen, W:: On multivariate B-splines. SIAM J. Numer. Anal. 17(2), 179-191 (1980)

29. de Boor, C., de Voer, R.: Approximation by smooth multivariate splines. Trans. Am. Math. Soc. 276(2), 131-148 (1983)

30. Cox, M.G.: The numerical evaluation of B-splines. IMA J. Appl. Math. 10(2), 134-149 (1972)

31. de Boor, C.: On calculating with B-splines. J. Approx. Theory 6(1), 50-62 (1972)

32. Hall, C.A., Meyer, W.W.: Optimal error bounds for cubic spline interpolation. J. Approx. Theory 16(2), 105-122 (1976)

33. Hall, C.A.: Natural cubic and bicubic spline interpolation. SIAM J. Numer. Anal. 10(6), 1055-1060 (1973)

34. Kolman, R., Okrouhlík, M., Berezovski, A., Gabriel, D., Kopačka, J., Plešek, J.: B-spline based finite element method in one-dimensional discontinuous elastic wave propagation. Appl. Math. Model. 46, 382-395 (2017)

35. Soliman, A.A.: A Galerkin solution for Burgers' equation using cubic B-spline finite elements. Abstr. Appl. Anal. 46, 382-395 (2012)

36. Bai, D.M., Zhang, L.M.: The quadratic B-spline finite element method for the coupled Schrodinger-Boussinesq equations. Int. J. Comput. Math. 88(8), 1714-1729 (2010)

37. Kutluay, S., Esen, A.: A B-spline finite element method for the thermistor problem with the modified electrical conductivity. Appl. Math. Comput. 156(3), 621-632 (2005)

38. Dag, I., Naci Ozer, M.: Approximation of the RLW equation by the least square cubic B-spline finite element method Appl. Math. Model. 25(3), 221-231 (2001)

39. Raslan, K.R.: A collocation solution for Burger's equation using quadratic B-spline finite elements. Int. J. Comput. Math 80(7), 931-938 (2003)

\section{Submit your manuscript to a SpringerOpen ${ }^{\circ}$ journal and benefit from:}

- Convenient online submission

- Rigorous peer review

- Open access: articles freely available online

- High visibility within the field

- Retaining the copyright to your article

Submit your next manuscript at $>$ springeropen.com 